\title{
Collaborative Multiple-Access Protocols for Wireless Networks
}

\author{
Ahmed K. Sadek， K. J. Ray Liu， and Anthony Ephremides \\ Department of Electrical and Computer Engineering, and Institute for Systems Research \\ University of Maryland, College Park, MD 20742, USA. \{aksadek, kjrliu, etony\} @eng.umd.edu
}

\begin{abstract}
In this paper, a new multiple access approach is proposed that takes into account the broadcast nature of the wireless channel. The new approach employs a relay to boost the system throughput. This approach is based on a new idea in which the relay utilizes the empty time slots available in a TDMA frame. The relay stores the packets that failed transmissions in previous time slots. At each time slot, the relay listens to the channel and retransmits the packet at the head of its queue if the channel is free. This will better utilize the channel resources and will introduce on-demand spatial diversity into the network. Two different protocols are proposed to implement this new multiple-access scheme. The stability criteria of the associated queueing systems are studied and analytical expressions for the maximum stable throughput are provided for the symmetrical users case. Numerical results indicate a significant increase in the maximum stable throughput by using the new multiple-access protocol over pure TDMA.
\end{abstract}

\section{INTRODUCTION}

Over the past few decades, wireless communications and networking have witnessed an unprecedented growth. The growing demands require very high data rate and considerably large coverage areas, especially for cellular networks. This poses challenges in the deployment of wireless networks. Increasing the transmitting power and/or increasing the number of base-stations (BS) or access points (AP) can provide solutions to this challenge. It is obvious, however, that these solutions are not preferable as the power of the wireless terminals is usually limited, and building more basestations or access points is costly.

Relay-based wireless networks have been discussed as possible solution for these future demands in [1]. R. Pabst et al. [1] have envisioned that incorporating fixed (or moving) relays in the network might be a necessary modification in the wireless network architecture to meet the desirable high throughput and coverage required for future applications. In recent standards, fixed relays (usually called wireless routers in the Mesh network terminologies) are a characteristic flavor of Mesh networks [2] which introduce a special hierarchy in wireless networks. This motivated us to study the implementation of new multiple-access protocols for relay-based

\footnotetext{
${ }^{1}$ This work was supported in part by U.S. Army Research Laboratory under Cooperative Agreement DAAD 190120011.
}

wireless networks. From the information theoretic perspective, the relay channel has been first addressed in the seminal work by Cover and El-Gamal [17]. Recently, the concept of cooperative diversity has gained a lot of interest [3], [4], [5], [6], [7], [8]. In cooperative diversity, one or more relays cooperate with a source node to help in forwarding its data to a destination. This can achieve spatial diversity as the data is transmitted via spatially independent channels. In [9], [10], Maric and Yates applied the concept of cooperation in multicasting and broadcasting to maximize the network life time.

In this paper, we study the employment of relays in implementing new multiple access schemes under a TDMA framework. We introduce a new approach for multiple access in which the relay utilizes the empty time slots in a TDMA frame to retransmit the failed transmitted packets. More specifically, the relay stores the packets that were not received correctly by the AP in its queue. At the beginning of each time slot, the relay listens to the channel and if the time slot is empty the relay transmits the packet at the head of its queue. This new multiple-access (MA) approach thus jointly designs the physical and MA layers in a cross-layer fashion. We propose two protocols $S^{1}$ and $S^{2}$ to implement this idea. In this work, we only consider the symmetric users case in which users have the same data arrival rates and channel statistics. We study the stability criteria for the corresponding system of queues and accordingly characterize the maximum stable throughput that can be achieved by these new MA protocols. The maximum stable throughput of one of the two proposed MA protocols significantly exceeds that of pure TDMA. This also translates into smaller overall system delay. We conjecture that the total stability region of TDMA is contained inside the stability region of protocol $S^{1}$, but we defer this study to a further work due to space limitations.

The rest of the paper is organized as follows. The system model is described in Section II. The new MA approach and the two proposed protocols are introduced in Section III. Numerical results for the theoretically calculated maximum stable throughput are addressed in Section IV, and finally conclusions are drawn in the same section. 


\section{SYSTEM MODEL}

We consider the uplink of a TDMA system in which $N$ users are trying to communicate with an AP or a BS. Time is slotted, and each user is assigned a certain time slot. We assume a symmetric finite user scenario $(N<\infty)$ with infinite buffer length and packets arrival rate with mean $\lambda / N$. The arrival process follows a Bernoulli process. The wireless channel between any two nodes in the network is modeled as a Rayleigh flat fading channel with additive Gaussian noise.

As mentioned in the Introduction, we consider the availability of a fixed relay/wireless router (WR) that belongs to the infrastructure of the network, and we study the utilization of this relay to enhance the system performance. The performance here is measured by the maximum stable throughput that can be achieved by the network. The maximum stable throughput can be defined as follows.

Definition 1: The maximum stable throughput $\lambda_{M S T}$ is defined as the supremum of the stable data arrival rates, i.e., the system is stable if and only if the data arrival rate $\lambda$ is smaller than $\lambda_{M S T}$. Stability will be rigorously defined later.

Next, we model the signal received at any receiving terminal. Denote the set of transmitting nodes by $\mathcal{T}=\{i, W R$ : $1 \leq i \leq N\}$, which consists of the $N$ users and the WR. Also denote the set of receiving nodes by $\mathcal{R}=\{W R, A P\}$ which consists only of the WR and AP. The signal received at a receiving end can be modeled as

$$
y_{i j}=\sqrt{P} h_{i j} x_{i}+n_{i j}, \quad i \in \mathcal{T}, j \in \mathcal{R}, i \neq j,
$$

where $P$ is the transmitting power assumed the same for all transmitting terminals, $h_{i j}$ is the channel gain between nodes $i$ and $j$ and is modeled as a zero mean, circularly symmetric complex Gaussian random variable. The variance of the channel gain takes values $\sigma_{s}^{2}, \sigma_{s r}^{2}, \sigma_{r}^{2}$ which corresponds to the user-AP, user-WR(relay), and WR(relay)-AP links, respectively. The term $x_{i}$ denotes the transmitted packet with unit power, and $n_{i j}$ denotes the additive white Gaussian noise with zero mean and variance $N_{o}$.

From (1), the received signal-to-noise ratio (SNR) of a signal transmitted between two terminals $i, j$ can be calculated as

$$
\mathrm{SNR}_{i j}=\frac{\left|h_{i j}\right|^{2} P}{N_{o}} .
$$

In this paper we characterize the success or failure of a packet reception by the outage event. Outage is defined as the event that the received SNR falls below a certain threshold $\gamma$, hence, the probability of outage $P_{O}$ is defined as,

$$
\mathcal{P}_{O}=\mathcal{P}\left(\mathrm{SNR}_{i j} \leq \gamma\right) .
$$

The SNR threshold $\gamma$ is determined according to the application and the transmitter/receiver structure. If the received SNR is higher than the threshold $\gamma$, the receiver is assumed to be able to decode the received message with negligible probability of error. If an outage occurs, the packet is considered lost. This is equivalent to the capture model with the difference that the transmitted packets do not encounter interference from other users in our system [14], [13]. From (2), the outage probability in (3) can be calculated as follows

$$
\mathcal{P}_{O}=1-\exp \left(-\frac{\gamma}{S N R}\right),
$$

where SNR $\in\left\{\mathrm{SNR}_{s}=\frac{P \sigma_{s}^{2}}{N_{o}}, \mathrm{SNR}_{s r}=\frac{P \sigma_{s r}^{2}}{N_{o}}, \mathrm{SNR}_{r}=\right.$ $\left.\frac{P \sigma_{r}^{2}}{N_{o}}\right\}$ according to the transmitting and receiving ends.

\section{Protocols Description}

In a pure TDMA system, if a user does not have a packet to transmit, his time slot remains idle, i.e., wasted channel resources. In the presence of a relay in the network, we can employ this relay to better utilize the channel resources. In this section, we introduce a novel multiple access approach based on employing relays in the wireless network. We describe two practical protocols to implement this novel approach and analyze their performance in terms of the maximum stable throughput. We will refer to these two protocols by $\mathcal{S}^{1}$ and $\mathcal{S}^{2}$.

First, we introduce the new multiple access approach. The relay, or WR, receives the packets transmitted by the users to the AP. In case of the event that the packet is not received correctly by the AP, the WR stores this packet in its queue, if the WR was able to decode this packet correctly. Hence, the WR's queue contains packets that have failed transmission. At the beginning of each time slot, the WR senses the channel to check whether the time slot is empty or not. If the time slot is empty the WR will retransmit the packet at the head of its queue, hence utilizing this channel resource that was previously wasted in pure TDMA. Moreover, this introduces spatial diversity in the network as the channel fades between different nodes in the network are independent. Next, we will introduce two protocols to implement this new idea and compare their performance in terms of the maximum stable throughput, and also compare their performance to that of TDMA.

\section{A. Protocol $S^{1}$ : Implementation and Analysis}

The main characteristic of protocol $S^{1}$ is that when a user has a failed transmission in his time slot, and given that the WR was not able to deliver this packet during the rest of the TDMA frame and the turn comes again to this user, then this user retransmits this failed packet again even if he/she has new packets waiting transmission. To better understand the operation of this protocol we summarize it in the following steps.

- Time is slotted and TDMA is utilized for multiple access.

- At the beginning of a time slot, if a user has a new packet to transmit and has no backlogged packets then the user 
transmits this packet to the AP. Due to the broadcast nature of the wireless channel, the WR can also receive the transmitted packet with certain success probability.

- If the packet is not received correctly by the AP, then the AP is going to feedback a negative acknowledgement (NACK) declaring the packet's failure. In this case, if the WR was able to receive the packet correctly then it stores this packet in its queue waiting for a retransmission.

- At the beginning of each time slot, the wireless router senses the channel to check if there is a transmission, if the channel is free then the wireless router transmits the packet at the head of its queue.

- At the beginning of a time slot, if the corresponding user has a failed transmitted packet, then the user is going to retransmit this packet even if he has new packets to transmit.

According to the above description of protocol $S^{1}$, the WR's queue can at most have $N$ backlogged packets, where $N$ equals the number of users in the system. This follows because according to the protocol, the WR can have at most one packet from each user. Therefore, the WR's queue never overflows and the stability of the system is mainly determined by the stability of the users' queues.

Next we calculate the maximum stable throughput of protocol $S^{1}$. Denote the size of the queues of the transmitting nodes, set $\mathcal{T}$, at any time $t$ by the vector $\mathbf{q}^{t}=$ $\left[q_{1}^{t}, q_{2}^{t}, \cdots, q_{W R}^{t}\right]$. We adopt the definition of stability used in [11]

Definition 2: Queue $i \in \mathcal{T}$ of the system is stable, if

$$
\lim _{t \rightarrow \infty} \mathcal{P}\left\{q_{i}^{t}<x\right\}=F(x) \quad \text { and } \quad \lim _{x \rightarrow \infty} F(x)=1 .
$$

If

$$
\lim _{x \rightarrow \infty} \lim _{t \rightarrow \infty} \inf \mathcal{P}\left\{q_{i}^{t}<x\right\}=1,
$$

the queue is substable.

From the definition, if a queue is stable then it is also substable. If a queue is not substable, then it is unstable.

The multidimensional stochastic process $\mathbf{q}^{t}$ is an irreducible and aperiodic Markov chain with countable number of states. According to the definition of stability, the Markov chain is stable if and only if there is a positive probability for every queue of being empty [16], i.e.,

$$
\lim _{t \rightarrow \infty} \mathcal{P}\left\{q_{i}(t)=0\right\}>0 .
$$

If the arrival and departure processes of a queueing system are strictly stationary, then one can apply Loynes's theorem to check for stability conditions [12]. This theorem states that, if the arrival process and the departure process of a queueing system are strictly stationary, and the average arrival rate is less than the average departure rate, then the queue is stable; if the average arrival rate is greater than the average departure rate then the queue is unstable. We will not consider the boundary conditions in this work.

The system of queues in $S^{1}$ are interacting. The reason behind this is the fact that serving the failed packets of a certain queue depends on how often the other queues empty. More specifically, if we just have two users, i.e. $N=2$, and one of the two users' queues was empty for a long time, then the WR serves the lost packets from the other user more often. On the other hand, if one of the two users queues never empties, then the other user will never get served by the WR. Studying stability conditions for interacting queues is a difficult problem that has been studied in the context of ALOHA systems, i.e., random access systems [15], [16]. Rao and Ephremides [15] introduced the concept of dominant systems to help finding bounds on the stability region of a system of interacting queues. This was studied in the context of random access, and the dominant system was defined in a way to help decouple the interacting queues. The dominant system in [15] was defined by allowing a set of users who have no packets to transmit to continue transmitting dummy packets. In our system $S^{1}$, we define the dominant system in a different way that suits the TDMA framework and the employed WR in order to help decouple the interaction of the queues and hence analyze the system performance. We define the dominant system for $S^{1}$ as follows. For $1 \leq j \leq N$, define $S_{j}^{1}$ as

- 1) Arrivals at queue $i$ in $S_{j}^{1}$ is the same as $S^{1}$.

- 2) The channel realizations $h_{k l}$, where $k \in \mathcal{T}$ and $l \in \mathcal{R}$, for both $S_{j}^{1}$ and $S^{1}$ are identical.

- 3) The noise generated at receiving ends of both systems are identical.

- 4) The WR does not serve the first $j$ queues in $S_{j}^{1}$.

Conditions 1, 2, and 3 guarantee that the decisions made at the receiving ends of both systems are identical, i.e., the sequence of failed packets detected at both systems are identical. Condition 4 means that the first $j$ queues act as in a TDMA system. The WR, however, can help the other users $j+1 \leq k \leq N$ in the empty slots of the TDMA frame.

Now consider system $S_{N}^{1}$ in which the WR does not help any of the users, i.e., pure TDMA. It is clear that the queue sizes in this system are never smaller than those in the original system $S^{1}$. For $S_{N}^{1}$, the success probability of transmitting a packet is given by ${ }^{1}$

$$
P_{s}\left(S_{N}^{1}\right)=\mathcal{P}(\mathrm{SNR} \geq \gamma)=\exp \left(-\frac{\gamma}{\mathrm{SNR}_{s}}\right)
$$

The service rate per user is thus given by

$$
\mu\left(S_{N}^{1}\right)=\frac{1}{N} \exp \left(-\frac{\gamma}{\mathrm{SNR}_{s}}\right),
$$

where $\frac{1}{N}$ is because the time is divided equally among the $N$ users. Since system $S_{N}^{1}$ acts as a pure TDMA system, the

\footnotetext{
${ }^{1}$ Note that in this paper we will only consider the dominant system $S_{N}^{1}$ as we are only interested in the symmetrical data arrival case.
} 
queues are decoupled and hence the arrival process and departure process of each of them is strictly stationary. Applying Loynes theorem, the stability condition for $S_{N}^{1}$ is given by $\lambda / N<\mu\left(S_{N}^{1}\right)$, or in other words

$$
\lambda<\exp \left(-\frac{\gamma}{\mathrm{SNR}_{s}}\right) .
$$

Next, let us consider the stability of $S^{1}$. Since $S_{N}^{1}$ as described before dominates $S^{1}$, if $S_{N}^{1}$ is stable then $S^{1}$ is also stable. Therefore, for $\lambda<\exp \left(-\frac{\gamma}{\mathrm{SNR}_{s}}\right)$ system $S^{1}$ is stable. On the other hand, if all the queues in $S_{N}^{1}$ are unstable, then none of these queues ever empty, hence, the WR loses its role and both systems $S^{1}$ and $S_{N}^{1}$ are indistinguishable. Therefore, if we have $\lambda>\exp \left(-\frac{\gamma}{\mathrm{SNR}_{s}}\right)$ then all the queues in $S_{N}^{1}$ are unstable and accordingly system $S^{1}$ is unstable. Therefore, the maximum stable throughput for system $S^{1}$ can be summarized in the following theorem.

Theorem 1: The maximum stable throughput $\lambda_{M S T}\left(S^{1}\right)$ for system $S^{1}$ is equal to that of a pure TDMA system and is given by

$$
\lambda_{M S T}\left(S^{1}\right)=\exp \left(-\frac{\gamma}{\mathrm{SNR}_{s}}\right) .
$$

Although the maximum stable throughput for system $S^{1}$ is equal to that of pure TDMA, we conjecture that the total stability region of a TDMA system is contained inside that of system $S^{1}$. We will study this in more details in a future work.

\section{B. Protocol $S^{2}$ : Implementation and Analysis}

The main difference between protocols $S^{1}$ and $S^{2}$ is in the role of the WR and the behavior of the users' regarding their backlogged packets. More specifically, we describe the implementation of $S^{2}$ in the following steps. For clarity of presentation, and without loss of generality, let us consider a test user who has a new packet to transmit and who does not have any backlogged packets

- At the beginning of this test user's time slot, the user will transmit the new packet he has and both the WR and the AP receive it. If the packet is received correctly by the AP then it sends back an ACK and the packet is released from both the WR's and the user's queues, otherwise the AP sends back a NACK. In case of the later event, if the WR was able to receive the packet correctly then it stores the packet in its queue and sends back an ACK.

- If an ACK is received back from either the AP or the WR, then the test user releases this packet completely from his queue.

- At the beginning of each time slot, the WR senses the channel to decide whether or not a new transmission is taking place. If not, then the WR transmits the packet at the head of his queue.
- In the next test user's time slot, if the test user has a new packet to transmit then he transmits this packet whether or not he has any backlogged packets stored at the WR.

It is clear from the above description of the protocol implementation that once any packet is received correctly by the $\mathrm{AP}$ and/or the WR, then the corresponding user forgets completely about this packet. The packets which have not been correctly received by the AP but correctly received by the WR are the WR responsibility. The WR serves these packets in the empty time slots of the users. One can now figure out the differences between the queues in system $S^{1}$ and $S^{2}$ :

- i) The WR's queue can grow without limit in $S^{2}$ as it can have more than one packet from each user, however, it can not exceed size $N$ in $S^{1}$.

- ii) The user's queues in $S^{2}$ are not interacting as the case in $S^{1}$. This is because the user forgets about the packets which were received correctly by the WR and they are released from his queue. In other words, servicing the queue of any user depends only on the channel conditions from that user to the AP and WR, and does not depend on the status of the other users' queues.

Now we study the stability of system $S^{2}$. System $S^{2}$ is stable if and only if the queues $\mathbf{q}^{t}=\left[q_{1}^{t}, q_{2}^{t}, \cdot, q_{N}^{t}, q_{W R}^{t}\right]$ are stable. This means that for stability we require both the $N$ users' queues and the WR's queue to be stable. Hence, in general, the stability region of the whole system is the intersection of the stability regions of the $N$ users and the WR. Since in this paper we are only interested in the maximum stable throughput, we will calculate the maximum stable throughput of the $N$ users' queues and the WR queue and find the minimum of both.

First, we calculate the maximum stable throughput of the $N$ users' queues. According to the operation of system $S^{2}$, a user succeeds in transmitting a packet if either the AP or the WR receives this packet correctly. The success probability of a user in $S^{2} P_{s, U}\left(S^{2}\right)$ can thus be calculated as

$$
P_{s, U}\left(S^{2}\right)=\mathcal{P}\left(E_{1} \cup E_{2}\right),
$$

where $E_{1}$ denotes the event that the WR received the packet successfully, and $E_{2}$ denotes the event that the AP received the packet successfully. Similar to the calculations of the success probability of system $S^{1}$, the success probability of the users in $S^{2}$ can be calculated as

$$
\begin{aligned}
P_{s, U}\left(S^{2}\right) & =\exp \left(-\frac{\gamma}{\mathrm{SNR}_{s}}\right)+\exp \left(-\frac{\gamma}{\mathrm{SNR}_{s r}}\right) \\
& -\exp \left(-\frac{\gamma}{\mathrm{SNR}_{s}}\right) \exp \left(-\frac{\gamma}{\mathrm{SNR}_{s r}}\right) .
\end{aligned}
$$

The maximum stable throughput of the $N$ users' queues in $S^{2}$ is thus given by

$$
\begin{aligned}
\lambda_{M S T}\left(S^{2}, U\right) & =\exp \left(-\frac{\gamma}{\mathrm{SNR}_{s}}\right)+\exp \left(-\frac{\gamma}{\mathrm{SNR}_{s r}}\right) \\
& -\exp \left(-\frac{\gamma}{\mathrm{SNR}_{s}}\right) \exp \left(-\frac{\gamma}{\mathrm{SNR}_{s r}}\right) .
\end{aligned}
$$


Now, we calculate the maximum stable throughput of the WR. The arrival rate to the WR depends on the packets transmitted by the users and not successfully received by the AP given that the WR was able to correctly receive them. Therefore, the arrival rate to the wireless router per TDMA frame is given by

$$
\lambda_{W R}=\frac{\lambda}{P_{s, U}\left(S^{2}\right)}\left(1-\exp \left(-\frac{\gamma}{\mathrm{SNR}_{s}}\right)\right) \exp \left(-\frac{\gamma}{\mathrm{SNR}_{s r}}\right),
$$

where $\lambda / P_{s, U}\left(S^{2}\right)$ accounts for the probability of having the users'queues not empty, i.e., has a packet to transmit; $\lambda$ is the total arrival rate for the $N$ users in the system. The service rate of the WR depends on when the $N$ users queues are empty. The probability of having a user empty is given by [18]

$$
\mathcal{P}(\text { empty user })=1-\frac{\lambda}{N \mu\left(S^{2}, U\right)},
$$

where $\lambda / N$ is the arrival rate per user, and $\mu\left(S^{2}, U\right)$ is the service rate per user and is given by

$$
\mu\left(S^{2}, U\right)=\frac{1}{N} P_{s, U}\left(S^{2}\right),
$$

where $P_{s, U}\left(S^{2}\right)$ is the success probability of a user and is defined in (13). Now, we calculate the average service rate of the WR. The WR services the lost packets in the empty time slots available, and this happens with rate

$$
\mu_{W R}=\left(1-\frac{\lambda}{P_{s, U}\left(S^{2}\right)}\right) \exp \left(-\frac{\gamma}{\mathrm{SNR}_{r}}\right)
$$

which is the average number of empty time slots per frame multiplied by the probability of successfully transmitting a packet from the WR to the AP. It could be shown that a sufficient condition for the stability of the WR's queue is that its arrival rate is less than its average success probability [15]. Hence, the maximum stable throughput for the WR satisfies the following inequality

$$
\begin{aligned}
\frac{\lambda}{P_{s, U}\left(S^{2}\right)}\left(1-\exp \left(-\frac{\gamma}{\mathrm{SNR}_{s}}\right)\right) & \exp \left(-\frac{\gamma}{\mathrm{SNR}_{s r}}\right)< \\
& \left(1-\frac{\lambda}{P_{s, U}\left(S^{2}\right)}\right) \exp \left(-\frac{\gamma}{\mathrm{SNR}_{r}}\right)
\end{aligned}
$$

Solving the above inequality, the maximum stable throughput for the WR can be given by

$$
\lambda_{M S T}\left(S^{2}, W R\right)=\frac{\exp \left(-\frac{\gamma}{\mathrm{SNR}_{r}}\right) P_{s, U}\left(S^{2}\right)}{\Phi},
$$

in which

$\Phi=\exp \left(-\frac{\gamma}{\mathrm{SNR}_{r}}\right)+\exp \left(-\frac{\gamma}{\mathrm{SNR}_{s r}}\right)\left(1-\exp \left(-\frac{\gamma}{\mathrm{SNR}_{s}}\right)\right)$ where $P_{s, U}\left(S^{2}\right)$ is given by (13).

Having calculated the maximum stable throughput of the users and WR in system $S^{2}$, given by (14) and (20), respectively, the maximum stable throughput of system $S^{2}$ is specified by the following theorem.

Theorem 2: The maximum stable throughput for system $S^{2}$ is determined by the minimum of the maximum stable throughputs of the users' queues and the WR as follows

$$
\lambda_{M S T}\left(S^{2}\right)=\min \left(\lambda_{M S T}\left(S^{2}, U\right), \lambda_{M S T}\left(S^{2}, W R\right)\right) .
$$

where $\lambda_{M S T}\left(S^{2}, U\right)$ is the maximum stable throughput of the users' queues and is specified in (14), and $\lambda_{M S T}\left(S^{2}, W R\right)$ is the maximum stable throughput of the WR and is specified in (20).

\section{NumERICAL RESUlts AND CONCLUSIONS}

We plot the analytical expressions derived for the maximum stable throughput for some practical systems values. The relay is taken at half the distance between the user and the $\mathrm{AP}$, and the propagation constant is equal to 3 . For a $N=10$ users system and for a SNR threshold of $\gamma=3,10 \mathrm{~dB}$, Fig. 1 depicts the comparison between the maximum stable throughput of the users' queues and the WR's queue. The figure reveals that the WR's queue is the bottleneck for system $S^{2}$ and not the users. Fig. 2 depicts the maximum stable throughput for systems $S^{1}$ and $S^{2}$. Note that system $S^{1}$ is identical to a TDMA system for the symmetrical case only. This figure reveals that the maximum stable throughput for system $S^{2}$ is significantly larger than that of system $S^{1}$ or equivalently TDMA. For some SNR values, there is a $150 \%$ increase in the Maximum stable throughput via employing system $S^{2}$ over $S^{1}$ or TDMA. Note also from Fig. 2 that by decreasing the SNR threshold to $3 d B$, which means a receiver with much more powerful signal processing capabilities, the gap between the maximum stable throughput of systems $S^{2}$ and pure TDMA reduces. This is due to the fact that by increasing the complexity of the receiver architecture or the applied signal processing and coding techniques in the signal design, the probability of losing a packet decreases, hence, reducing the role of the relay. This can be thought of as a tradeoff between the MA and physical layer capabilities which emphasizes the importance of the cross-layer design framework for wireless networks..

In this paper, we have introduced a new approach for designing multiple-access protocols for relay-based wireless networks. The new approach employs the relay in utilizing the empty time slots in a TDMA frame to forward the packets failed in previous transmissions. This improves the utilization of channel resources which are otherwise wasted under a pure TDMA framework. The proposed approach also provides spatial diversity by allowing spatially separated terminals in forwarding the data. We proposed two practical 


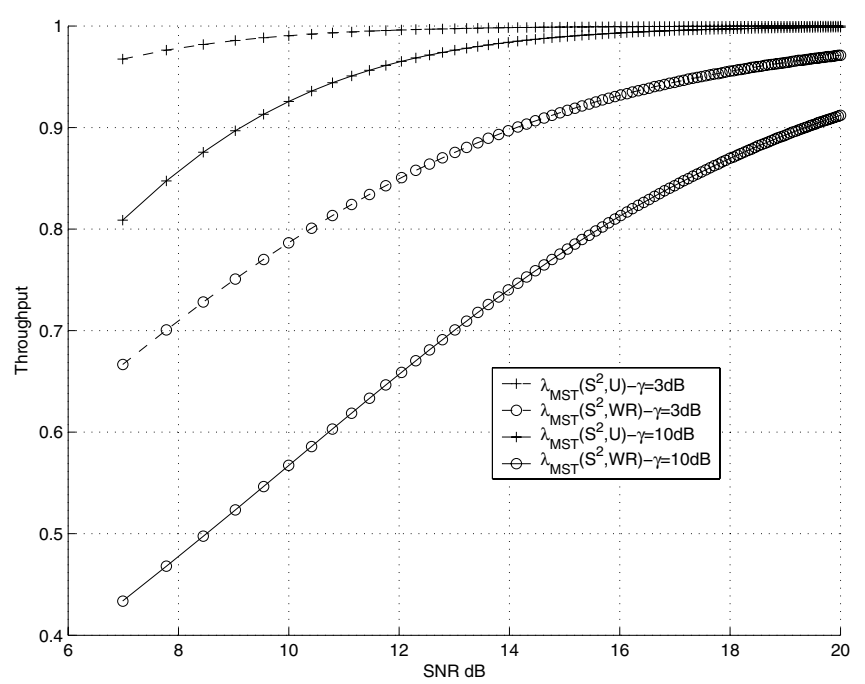

Fig. 1. Maximum stable throughput for the two systems of queues in protocol $S^{2}$ : the users' queues and the WR queue. The horizontal axis denotes the signal-to-noise ratio. The number of users equals 10 and $\gamma=0,10 \mathrm{~dB}$ The WR queue is the bottleneck for the whole system throughput as can be deduced from the curves.

protocols $S^{1}$ and $S^{2}$ to implement the new idea. We also analyzed the stability of the queueing systems under both protocols and provided analytical expressions for the maximum stable throughput that is achieved by each protocol. Numerical results indicate a significant increase in the maximum stable throughput by applying one of the new protocols over pure TDMA.

In this work, we only considered the symmetrical users' case in which users have the same data arrival rate and the same channel statistics. Future work include the study of asymmetric data arrival rates and channel statistics. This study will lead into characterizing the stability region of the proposed approach. At this point we conjecture that the stability region of pure TDMA is contained in the stability region of protocol $S^{1}$.

\section{REFERENCES}

[1] R. Pabst et al., "Relay-based deployment concepts for wireless and mobile broadband radio", IEEE Communications Magazine, vol. 42, no. 9, pp. 80-89, Sep. 2004.

[2] R. Bruno, M. Conti, and E. Gregori,"Mesh Networks: Commodity Multihop Ad Hoc Networks,", IEEE Communications Magazine, pp. 123131, March 2005.

[3] A. Sendonaris, E. Erkip, and B. Aazhang, "User cooperation diversityPart I: system description," IEEE Trans. Comm., vol. 51, pp.1927-1938, Nov. 2003.

[4] A. Sendonaris, E. Erkip, and B. Aazhang, "User cooperation diversityPart II: implementation aspects and performance analysis," IEEE Trans. Comm., vol. 51, pp.1939-1948, Nov. 2003.

[5] J. N. Laneman, D. N. C. Tse, and G. W. Wornell, "Cooperative diversity in wireless networks: efficient protocols and outage behavior," IEEE Trans. Inform. Theory, vol. 50, pp.3062-3080, Dec. 2004.

[6] J. N. Laneman and G. W. Wornell, "Distributed space-time coded protocols for exploiting cooperative diversity in wireless networks," IEEE Trans. Inform. Theory, vol. 49, pp.2415-2525, Oct. 2003.

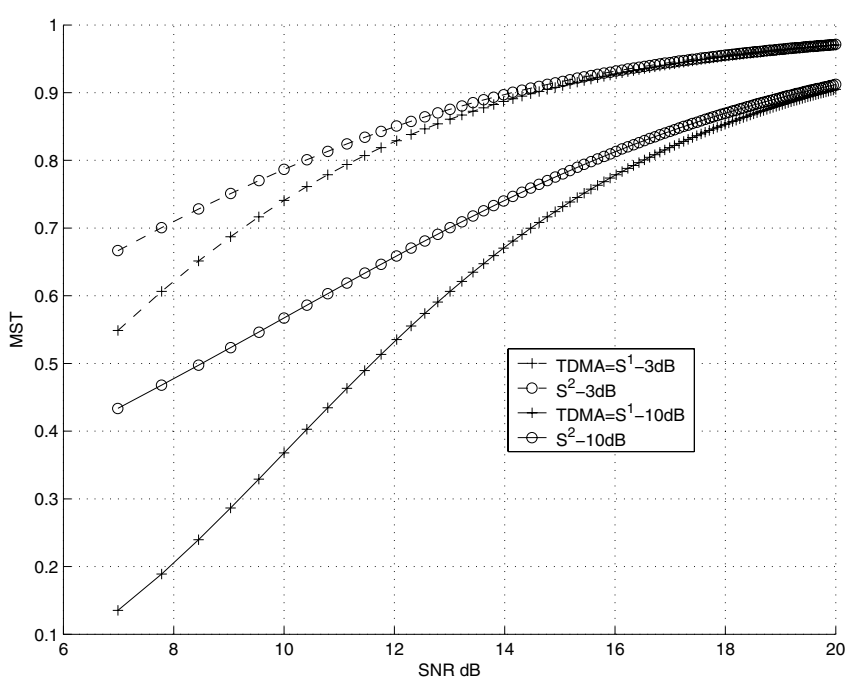

Fig. 2. Maximum stable throughput versus SNR for $S^{1} \equiv$ TDMA and $S^{2}$ for different SNR thresholds.

[7] W. Su, A. K. Sadek, and K. J. R. Liu, "SER performance analysis and optimum power allocation for decode-and-forward cooperation protocol in wireless networks," in proc. IEEE Wireless Communications and Networking Conference (WCNC'05), New Orleans, LA, March 13-17, 2005.

[8] A. K. Sadek, W. Su, and K. J. R. Liu, "Performance analysis for multinode decode-and-forward relaying in collaborative wireless networks," in proc. IEEE International Conference on Acoustics, Speech, and Signal Processing (ICASSP), Philadelphia, PA, March 19-23, 2005.

[9] I. Maric and R. Yates, "Cooperative multicast for network lifetime maximization," In Proceedings of the 42nd Allerton Conference on Communications, Control, and Computing, Oct. 2004.

[10] I. Maric and R. Yates, "Cooperative broadcast for maximum network lifetime," In Conference on Information Sciences and Systems CISS, Mar. 2004.

[11] W. Szpankowski, "Stability conditions for some multiqueue distributed systems: Buffered random access systems," Adv. Appl. Probab., vol. 26 , pp. 498-515, 1994.

[12] R. M. Loynes, " The stability of a queue with non-independent interarrival and service times," Proc. Cambridge Philos. Soc. 58, pp. $497-$ 520,1962

[13] M. Zorzi and R. Rao, "Capture and retransmission control in mobile radio," IEEE JSAC, vol. 12, pp. 1289-1298, Oct. 1994.

[14] B. Hajek, A. Krishna, and R. O. LaMaire, "On the capture probability for a large number of stations," IEEE Trans. on Communications, vol. 45, no. 2, pp. 254-260, Feb. 1997.

[15] R. Rao and A. Ephremides, "On the stability of interacting queues in a multi-access system," IEEE Trans. Inform. Theory, vol. 34, pp. 918-930, Sept. 1988.

[16] W. Luo and A. Ephremides, "Stability of $N$ interacting queues in random-access systems," IEEE Trans. Inform. Theory, vol. 45, no. 5, pp. 1579-1587, July 1999 .

[17] T. Cover and A. E. Gamal, "Capacity theorems for the relay channel," IEEE Trans. Inform. Theory, vol. 25, no. 5, pp. 572-584, Sept. 1979.

[18] D. Bertsekas and R. Gallager, "Data Networks," 2nd edition 